\title{
Intelligibilité mutuelle et contexte partagé
}

Inspirations conceptuelles et réductions technologiques

Mutual intelligibility and shared context. Conceptual inspirations and technological reductions

\section{Pascal Salembier et Moustapha Zouinar}

\section{OpenEdition}

\section{Journals}

Édition électronique

URL : http://journals.openedition.org/activites/1243

DOI : 10.4000/activites. 1243

ISSN : 1765-2723

Éditeur

ARPACT - Association Recherches et Pratiques sur les ACTivités

\section{Référence électronique}

Pascal Salembier et Moustapha Zouinar, «Intelligibilité mutuelle et contexte partagé », Activités [En ligne], 1-2 | octobre 2004, mis en ligne le 01 octobre 2004, consulté le 30 septembre 2020. URL : http:// journals.openedition.org/activites/1243; DOI : https://doi.org/10.4000/activites.1243

\section{(c) (†) $\ominus$}

Activités est mis à disposition selon les termes de la licence Creative Commons Attribution - Pas d'Utilisation Commerciale - Pas de Modification 4.0 International. 


\title{
Intelligibilité mutuelle et contexte partagé
} Inspirations conceptuelles et réductions technologiques

\author{
Pascal Salembier \\ GRIC-IRIT, UMR 5505 CNRS \\ Université Paul Sabatier \\ 118 , rte de Narbonne, \\ 31062 Toulouse Cedex \\ salembier@irit.fr
}

\author{
Moustapha Zouinar ${ }^{1}$ \\ GRIC-IRIT, UMR 5505 CNRS \\ Université Paul Sabatier \\ 118, rte de Narbonne, \\ 31062 Toulouse Cedex \\ moustafa.zouinar@rd.francetelecom.com
}

\begin{abstract}
RÉSUMÉ :
Historiquement, les cadres théoriques mobilisés pour donner une assise conceptuelle au programme technologique de conception de systèmes informatiques d'aide à la coopération homme-homme se réfèrent majoritairement au courant de l'«action située» et conséquemment à une de ses sources d'inspiration principale l'ethnométhodologie. Considérer sérieusement cette orientation nécessite de dépasser le stade du simple recours à des études empiriques ethnographiques et à repérer plus précisément les notions spécifiques structurantes de ce champ disciplinaire. Nous considérerons, dans cet article, l'influence de la notion de " descriptibilité » dans les recherches menées sur la coopération et son instrumentation. En contrepoint, nous présenterons une autre approche centrée sur la notion de " contexte partagé », qui se rapproche de certaines des orientations présentes dans le concept de descriptibilité mais qui s'en éloigne notablement sur d'autres.
\end{abstract}

MOTS-CLÉS :

Coopération ; Action et Cognition situées; Descriptibilité ; Contexte partagé ; Conception

\begin{abstract}
:
Mutual intelligibility and shared context. Conceptual inspirations and technological reductions

Historically, theoretical frameworks that are used in order to provide a conceptual base to the computer-supported cooperative work refer mainly to the "situated action" approach, and consequently to one of its main sources of inspiration: ethnomethodology. Taking this orientation seriously requires going beyond simply using ethnographically oriented field studies, and to identify precisely the specific notions which structure this disciplinary field. In this paper, we stress the influence of the notion of "accountability" in the works undertaken on cooperation and its technical support. In contrast, we present an alternative approach based on the notion of "shared context" which draws upon some of the orientations
\end{abstract}

1. Adresse actuelle : France Telecom R\&D, Issy-les-Moulineaux. 
that can be found in "accountability", but which exhibits some major differences.

KEYWORDS :

Cooperation; Situated action and cognition; Accountability; Shared context ; Design

\section{1.- Introduction}

Les activités coopératives finalisées font depuis plusieurs années l'objet d'un intérêt renouvellé. Bénéficiant de la caisse de résonnance technologique fournie par le développement rapide du courant CSCW (Computer-Supported Cooperative Work) et alimentés par la mutiplication d'études naturalistes menées dans les situations de travail, les travaux récents sur la coopération ont permis un traitement plus systématisé de la thématique. Ils ont notamment donné lieu à un effort de problématisation et de mise en discussion de notions jusque là peu abordées dans ce contexte (rôle des objets, distinction de registres de participation, accès partagé aux informations et aux ressource de l'environnement). Cette réflexion a bénéficié d'une part de la croissance d'un corpus général de données empiriques de plus en plus riche et d'autre part de travaux théoriques qui visent à fournir un cadre général de description et d'interprétation des activités coopératives, ainsi que des éléments d'orientation pour la conception de situations d'interaction coopérative médiatisée par des artefacts technologiques.

Ce renouveau d'intérêt pour les activités collectives porté par le CSCW ne signifie bien évidemment pas que celui-ci a investi un champ de recherches totalement vierge : si l'on se réfère uniquement par exemple à la psychologie du travail et à l'ergonomie de tradition francophone la liste est longue des travaux qui ont, d'une façon ou d'une autre, abordé ce thème (Leplat, 1991), en convoquant parfois des arrière-plans théoriques récemment éclairés des feux d'une modernité redécouverte ${ }^{1}$. Il reste néanmoins qu'en termes de visibilité et de diffusion, les recherches récentes sur la coopération ont largement bénéficié de l'importance des enjeux technologiques, organisationnels et économiques associés au CSCW. Un autre facteur de visibilité de ces travaux tient sans doute également au fait que la plupart des cadres théoriques mobilisés se positionnent dans un rapport d'opposition plus ou moins radical au paradigme cognitiviste dominant, ce qui a facilité leur diffusion dans le contexte d'une remise en question scientifique de plus en plus pressante de ce dernier.

Les sources d'inspiration conceptuelles sur lesquelles le CSCW s'efforce de construire ses fondements théoriques sont diverses ${ }^{2}$. Il est néanmoins généralement admis qu'historiquement, l'influence la plus notable est à chercher du côté de la sociologie et plus précisément de sa déclinaison ethnométhodologique (Harper, \& Hughes, 1993 ; Heath, \& Luff, 1992, 2000) qui fonde l'hypothèse de l'« action située ». Originellement le courant de l'« action située » trouve donc ses racines dans les travaux de Garfinkel sur la caractérisation des propriétés indexicales de l'action pratique et dans l'utilisation ultérieures de ces travaux par Lucy Suchman dans le cadre de son analyse critique du programme cognitiviste (métaphore de l'Homme comme système de traitement de l'information). Plusieurs points de l'argumentaire de Suchman (limitation du rôle fonctionnel des plans comme structures de contrôle de l'action, importance donné à l'indexicalité et au contexte, et remise

1. Voir par exemple les études déjà anciennes de Savoyant (Savoyant, 1984) qui utilisaient les concepts de la psychologie soviétique bien avant la vogue actuelle pour les théories néo-vygostkiennes de l'activité (même si l'influence de Leontiev est d'ailleurs plus évidente dans les travaux de Savoyant que celle de Vygotsky).

2. Outre l'"action située », on citera également comme autres références privilégiées la "Théorie de l'Activité » (Kuutti, 1996 ; Engeström, Miettinen, \& Punamaki, 1999 ; Engeström, 2000) et la Cognition distribuée (Hutchins, 1995). Pour une présentation de la contribution de ces approches à l'étude de la coopération voir http://www-sv.cict.fr/cotcos/pjs/ 
en question de la notion de représentation interne, corrélative des deux points précédents (Visetti, 1989 ; Salembier, 1996) ont été repris par des auteurs issus de champs disciplinaires différents (Agre, 1993; Clancey, 1997) qui ont insisté sur les aspects les plus «individualistes»de l'approche située : remise en cause des plans comme représentations internes intégralement prescriptives de l'action et « sensibilité cognitive » au contexte. C'est cette lecture de l' «action située » qui est sans doute la mieux connue en psychologie et en ergonomie cognitive ; mais dans le même temps elle a donné lieu à des reformulations dites « faibles » des positions principielles de l' « action située » et est à l'origine de malentendus persistants sur la réalité que recouvre le terme «situé » : confusion pointée (Quéré, 2000 ; Luff, \& Heath, 2000) entre « situé » et « en situation », et amalgame entre position critique de l'«action située » sur le rôle fonctionnel des plans et certains modèles représentationnalistes de planification opportuniste.

La sur-représentation de la critique des plans dans les débats qui ont suivi la publication de l'ouvrage de Suchman (Cognitive Science, 1993) a contribué à reléguer au second plan un aspect fondamental, déjà présent dans les travaux précédents de Suchman et plus généralement dans le champ de l'ethométhodologie : l'accent mis dans les interactions sur les processus de production d'intelligibilité mutuelle et d'accès partagé aux ressources de l'environnement. Du point de vue de l'interaction les présupposés de l'«action située » peuvent en effet être formulés de la façon suivante (Salembier, Theureau, Zouinar, \& Vermersh, 2001) :

- la réussite de l'interaction repose sur une production de l'intelligibilité mutuelle, production favorisée par l'accès mutuel aux ressources disponibles dans un environnement matériel, social et culturel particulier et plus ou moins stabilisé ${ }^{1}$;

- les significations portées par le langage mis en œuvre au cours de cette production de l'intelligibilité mutuelle ne reposent pas seulement sur une interprétation des unités linguistiques utilisées, mais aussi sur une interprétation d'autres éléments de la situation.

Dans cet article nous nous intéresserons plus particulièrement au premier point en le reliant à deux concepts heuristiquement également intéressants mais reposant sur des arrière-plans théoriques très différents : d'une part le concept d'"accountability" qui occupe une place centrale dans les travaux ethnométhodologiques et d'autre part la notion de «contexte partagé » auquel il est fréquemment fait reférence en psychologie du travail et en ergonomie sous des termes différents.

Nous insisterons par ailleurs sur le fait que l'intelligibilité mutuelle est directement liée au problème du contrôle de l'activité, abondamment discuté par l'«action située » dans son analyse critique du plan comme représentation interne. Nous proposerons enfin une approche du partage d'informations contextuelles et de ses conditions de possibilité, orienté vers le projet technologique d'évaluation de l'efficacité des environnements de travail.

\section{2.- Le contrôle ${ }^{2}$ de l'activité : externalisation et distribution}

La position classique défendue en sciences cognitives pose que l'acteur est le siège unique du contrôle de l'action, selon un mode délibératif de prescription intégrale fondé sur les plans. Pour l'«action située » l'acteur partage ce contrôle avec plusieurs instances qui peuvent être des objets, des artefacts technologiques ou organisationnels, c'est-à-dire pour reprendre la terminologie latou-

1. Ce point précis ouvre sur des questions importantes (sur le plan théorique et technologique) mais hors du champ de cet article ; par exemple : la relation souvent mal spécifiée entre environnement, contexte et situation, et les conditions de production de l'intelligibilité mutuelle dans les interactions à distance.

2. L'utilisation du terme « contrôle » en soit pose problème. Sa signification pourra en effet être orientée différemment selon qu'on l'utilise dans un contexte cognitiviste (avec par conséquent une influence cybernétique non négligeable), ou dans un contexte plus marqué par les sciences sociales. 
rienne, des «non-humains » ${ }^{1}$, mais également les autres acteurs présents dans l'environnement. Le problème posé ici est donc celui de l'externalisation des structures de contrôle de l'activité dans des supports matériels (les objets) et organiques (les autres acteurs) et conséquemment des conditions de cette externalisation et des spécificités fonctionnelles de ces différents supports. Nous évoquerons ici trois types de supports possibles : les objets, les outils organisationnels (procédures, ...) et les autres acteurs

\section{1.- Le contrôle par les objets}

Deux modes de contrôle par les objets ont été documentés et discutés dans la littérature : le premier met l'accent sur les propriétés physiques et notamment morphologiques de l'objet qui vont donner perceptuellement des éléments d'information sur ses fonctions possibles (en faisant donc l'économie d'opérations de représentation et de traitement symbolique). On lit parfaitement ici l'influence des travaux de Gibson sur les affordances.

Un second mode de contrôle a trait à l'organisation spatiale de l'environnement (Kirsh, 1995 ; Conein, 1997). L'idée développée ici est que la manière dont on dispose des objets sur un espace de travail est susceptible de fournir des informations sur l'état du processus en cours mais également des éléments de guidage local de l'action sur ce qu'il est possible et, le cas échéant, nécessaire de faire : «l'arrangement spatial résultant de la disposition des objets instancie une séquentialité temporelle $»^{2}$ et allège ainsi les traitements internes de gestion du contrôle de l'activité. Cette hypothèse a été empiriquement documentée dans des situations très variées qu'elles soient professionnelles (le travail de bureau), domestiques (la réalisation d'une recette de cuisine, le montage d'une bicyclette) ou ludiques (l'utilisation d'une version informatique du jeu Tetris) (Conein, 1990 ; Kirsh, 1995).

Dans le domaine du contrôle aérien un objet particulier, le strip papier, a donné lieu à un grand nombre d'analyses au cours des dernières années (Harper, Hughes, \& Shapiro, 1989 ; Hughes, Randall, \& Shapiro, 1992 ; Bressolle, 1993 ; Salembier, 2002). Le strip appartient à la catégorie plus générale des « artefacts cognitifs» (Norman, 1991) : il s'agit d'un dispositif artificiel conçu pour préserver, afficher ou effectuer des opérations sur une information de façon à satisfaire une fonction représentationnelle ». Le strip permet en effet d'afficher des informations issues du système informatique de traitement des plans de vol, et de recevoir des annotations symboliques manuscriptes. Il présente également des caractéristiques physiques qui en font un « objet manipulable »: il peut être déplacé sur le tableau de strips dont l'organisation fournit alors une représentation de l'état du trafic (nombre d'avions devant être gérer simultanément, séquencement des vols, positionnement relatif dans l'espace contrôlé). Mais l'agencement des strips fournit également une information procédurale relatives aux actions à mettre en oeuvre (transfert d'un vol au secteur suivant, instruction de navigation pour écarter deux vols, ...)

1. Voir par exemple (Latour, 1992)

2. Conein, op.cit., p.30 


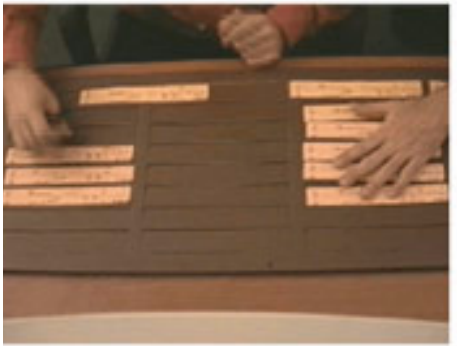

1

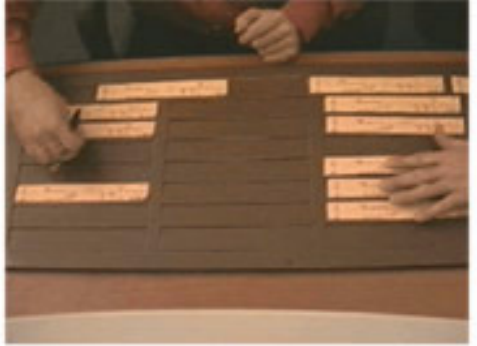

2

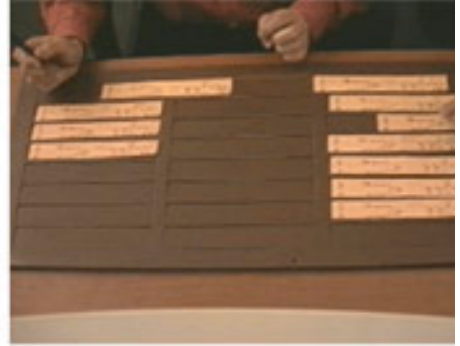

3

Figure 1.- Séquence de gestion collective du tableau de strips.

Le tableau de strips fournit une représentation externe, publiquement accessible de l'état du processus à un moment donné ; il permet donc la prise d'information incidente par les différents opérateurs co-présents dans l'environnement de travail. Il constitue également un lieu de rencontre des activités des contrôleurs (généralement deux) qui travaillent conjointement sur la position. Il permet ainsi de mettre en œuvre des routines et des stratégies de gestion collective du trafic.

Le strip remplit donc clairement une fonction d'assistance à l'activité individuelle (mémorisation, planification). L'identification de ses fonctions cognitives a d'ailleurs été utilisée pour spécifier et concevoir des outils d'assistance à l'activité qui s'inspire des propriétés des strips papier tout en les augmentant numériquement (Mertz, 2003). Mais il convient d'ajouter qu'en tant que représentation externe publiquement accessible le strip fournit également un support à la coordination de l'activité des différents opérateurs travaillant sur et autour de la position de contrôle (Harper et al., 1989; Bressolle, 1993) (figure 1).

\section{2.- Le contrôle par les artefacts organisationnels normatifs}

Par artefacts organisationnels normatifs nous entendrons ici essentiellement les procédures, les consignes, les guides de pratiques, les modèles processuels d'intervention, ... que Schmidt par exemple a rassemblés sous le terme générique de « constructs organisationnels formels » (Schmidt, 1997) ${ }^{1}$. Il peut paraître singulier de faire reférence dans le contexte de l'«action située » à ce type de constructs comme instances de contrôle de l'activité. En effet, concrétisant la perspective théorique de remise en question du rôle causal des plans dans l'accomplissement de l'action (et d'une certaine façon en écho à la tradition psychologique et ergonomique francophone), plusieurs études empiriques menées dans des situations professionnelles se sont attachées à mettre en évidence la sousdétermination des activités des opérateurs par ces artefacts organisationnels (Suchman, 1983 ; Suchman, \& Wynn, 1984 ; Button \& Sharrock, 1998). L'idée principale est ici que ces constructs sont utilisés comme des ressources (des «cartes », Schmidt, op.cit.) pour l' «action située » et non pas comme des systèmes de prescription intégrale. Certains n'ont d'ailleurs pas hésité à franchir le pas (de trop) en affirmant que ces constructs ne jouaient aucun rôle sur la détermination de l'action, ce qui bien évidemment relève de la pure «mystification » (Schmidt, 2000) : la question n'est pas ici de savoir si ces constructs jouent un rôle ou non sur la conduite de l'activité, mais d'identifier la façon dont ils sont utilisés, détournés, reconfigurés par les opérateurs dans la réalisation de leur travail.

Ainsi, dans des domaines d'activités dits «à risque ( (pilotage d'avions, conduite de centrales nucléaires, contrôle du trafic aérien, surveillance médicale des fonctions vitales, ...) où le moindre

1. Schmidt a ensuite décliné cette notion en « artefacts normatifs de coordination » dans le contexte du CSCW (Schmidt, 2000). 
écart à la prescription est considérée comme une source potentielle d'incident/accident, la réponse organisationnelle a souvent été pensée en termes de «sur-procéduralisation » de l'activité. Enoncer dans ce contexte que les procédures n'entretiennent aucune relation de détermination avec l'action apparaît comme une vue de l'esprit. Encore faut-il s'entendre sur le sens à donner à ce rapport de détermination ; dans une perspective " action située » on pourra dire avec Schmidt (en reférence à Wittgenstein) que cette forme de détermination n'est pas causale (elle ne cause pas l'action) mais normative (elle fournit une norme, une reférence pour l'accomplissement de l'action). Cette manière de concevoir les constucts formels et, au-delà, la nature de la détermination, permet ainsi d'élargir le champ des descripteurs et des dimensions pertinentes de l'activité. On peut alors concevoir que le suivi apparent de la procédure n'épuise pas l'activité des opérateurs. C'est ce qu'illustre par exemple très bien une étude menée dans le domaine du nucléaire par Theureau et al. (Theureau, Filippi, Saliou, \& Vermersch, 2002) : les auteurs y montrent de manière précise que le suivi efficace d'une procédure requiert un « habitus » de lecture de consignes qui n'est pas inscrit dans la procédure ; d'où l'analogie avec le suivi de partition musicale qui nécessite interprétation, correction et compléments pour parvenir à un résultat satisfaisant. En outre ce travail de « lecture-partition » s'entremèle avec d'autres actions qui répondent aux variations de l'environnement et aux sollicitations éventuelles des autres opérateurs et qui vont de ce fait entrainer des ruptures temporaires de l'activité de suivi de la procédure.

\section{3.- Le contrôle par les autres agents}

Les comportements des autres acteurs dans la situation constituent également une base d'orientation de l'activité :

«Pour un grand nombre de situations professionnelles et organisationnelles dans lesquelles les acteurs sont engagés, ce sont l'action et l'activité des tiers qui fournissent l'orientation principale dans la production pratique de la conduite, et ce sont les contributions de ces tiers qui définissent les contingences que les acteurs doivent gérer ». (Luff, \& Heath, 2000, p. 672, notre traduction ${ }^{1}$ ).

Ce contrôle peut s'opérer « naturellement » via des demandes explicites au travers d'actes de communication directs ou indirects, qui vont mettre en oeuvre des supports de médiation variés (le langage oral ou écrit, les gestes, les postures, ...). Ce contrôle peut également prendre une forme que l'on pourrait qualifier de non-intentionnelle, en ce que le comportement qui véhicule le contrôle n'est pas spécifiquement, consciemment adressé à une ou plusieurs personnes mais simplement rendu publique et donc potentiellement accessible à l'ensemble des agents co-présents dans l'environnement. Ces deux formes de contrôle s'entrelacent dynamiquement dans le cours de l'activité des agents : en fonction des propriétés structurelles de l'environnement de travail (arrangement spatial des postes par exemple) et des contingences de la situation (survenue d'un événement qui nécessite un traitement immédiat, disponibilité des opérateurs à un moment donné), une forme sera durablement ou ponctuellement prééminente. Un bon exemple de cette dynamique est donné par l'étude conduite par Suchman dans la salle des opérations d'une compagnie aérienne dans laquelle les opérateurs doivent coordonner l'ensemble des activités au sol requises par les départs et les arrivées des vols (Suchman, 1996).

\section{4.- Contrôle, coopération et accès conjoint aux ressources pour l'action}

Pour l'" action située » la coordination des activités n'est jamais totalement pré-spécifiée : c'est un processus de co-construction qui passe par la production d'intelligibilité mutuelle et par la vérification des conditions de cette intelligibilité.

1. NB : Sauf indication contraire, tous les extraits des textes cités dans cet article ont été traduits par nos soins. 
Une condition fondamentale de la production d'intelligibilité mutuelle tient à l'externalisation des représentations, des actions, et de leurs supports, qui va permettre un accès conjoint à ces ressources. De ce point de vue, la situation idéale c'est la co-présence physique d'agents coopérants ; c'est pourquoi Suchman dans son étude sur l'utilisation collaborative d'un photocopieur « intelligent » en fait la situation quasi-archétypale de la coopération. Une des raisons principales tient au fait que la coprésence permet en théorie un accès généralisé à l'ensemble des ressources qui supportent le processus d'interaction. Elle permet en outre la coordination des activités du collectif sur un mode plus économique que celui de la communication verbale, via des modes d'interaction non-intrusifs.

Plusieurs analyses empiriques menées dans des situations diverses ont illustré le rôle de l'accès des agents à l'activité des autres agents dans la gestion collective des activités et de dimensions plus spécifiques telles que la charge de travail, la répartition dynamique des tâches ou l'insertion d'actions ponctuelles dans un cours d'activité collectif. Cette gestion repose sur différents types d'information: les communications verbales, les gestes mais aussi des indices tels que les postures (Suchman, 1996 ; Zouinar, 2000 ; Heath, Sanchez-Svensson, Hindmarsh, Luff, \& vom Lehn, 2002).

Dans ces études l'information concernant l'activité des opérateurs est souvent partagée de façon qu'on pourrait qualifier d'incidente. Les actions ont été perçues du simple fait qu'elles étaient, de par les caractéristiques de l'environnement, accessibles aux différents agents. Il n'y a pas d'intention consciente de rendre observable une suite d'actions particulière. Dans d'autres situations par contre les opérateurs peuvent être amenés à rendre délibérément et sélectivement publique leur activité à destination de leurs collègues de façon à les informer d'un élément potentiellement pertinent pour eux. Heath et ses collègues ont donné plusieurs descriptions de ces pratiques dans des situations telles que la salle de contrôle du métro londonien (Heath, \& Luff, 1992) ou la salle de rédaction des dépêches d'une agence de presse (Heath et al., 2002).

C'est cet aspect de l'intelligibilité mutuelle que nous illustrerons dans l'exemple suivant tiré de nos travaux dans le contrôle aérien; il met en scène deux contrôleurs (CO et $\mathrm{CR}$ ) travaillant sur deux positions de contrôle adjacentes (TC et TE).

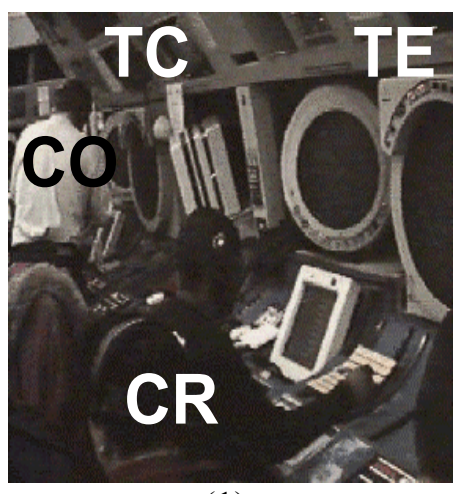

(1)

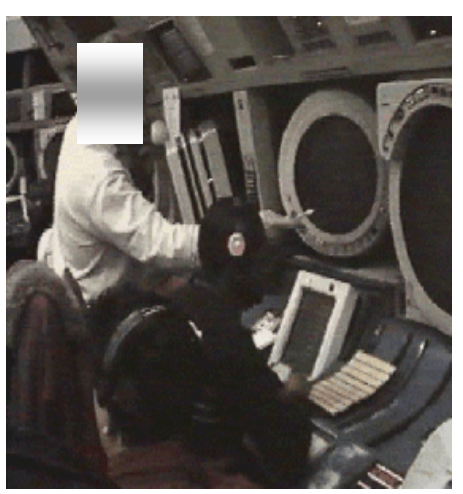

(2)

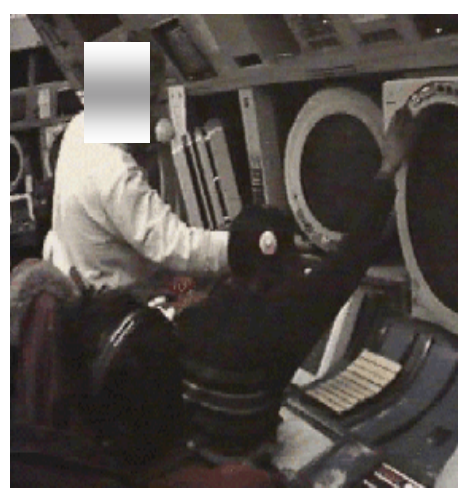

(3)

Figure 2.- Exemple d'intégration d'un tiers à une interaction entre deux participants principaux. Le contrôleur organique $(\mathrm{CO})$ de la position TC rend en partie audible au contrôleur radar de la position TE le contenu d'une communication téléphonique qui le concerne indirectement.

Le contrôleur organique (CO) du secteur TC est en communication téléphonique avec un interlocuteur sur un site distant (1). A un moment donné alors qu'il est toujours au téléphone il se dirige sur la position TE afin de rendre audible le contenu de sa conversation au CR-TE, de façon à ce que celuici puisse prendre connaissance de certains éléments de la situation et éventuellement ajuster son 
activité (2). Cette façon de procéder permet au CO-TC d'intégrer le CR à la situation d'interaction téléphonique avec un statut de «participant secondaire» (side-participant; cf. la classification introduite par Goffman) ponctuellement ratifié et donc de favoriser le partage d'une information pertinente tout en évitant d'interrompre la communication avec son interlocuteur. Le CR-TE manifeste qu'il a compris l'intention de son collègue en modifiant le réglage d'échelle de sa visu radar afin de mieux visualiser la situation du vol auquel le CO se réfere (3). L'intelligibilité de cette action est donc ici inscrite non seulement dans le cours d'activité dans lequel est engagé CR-TE mais également dans la référence au cours d'activité de CO-TC.

Ce mode de diffusion d'information peut prendre d'autres formes : ainsi en est-il des commentaires à haute voix qu'un agent produit « pour lui-même » dans le cours de son activité sans qui'il y ait forcément d'interlocuteur spécifique. En procédant ainsi il ne requiert pas de réponse obligée de la part de ses collègues et n'interrompt donc pas leurs tâches en cours; il rend néanmoins accessibles des éléments potentiellement utiles sur sa propre activité et/ou sur son évaluation de la situation en cours. Ce type de mécanismes, très souvent mis en œuvre dans les environnements de type salle de contrôle, joue un rôle important dans les situations de gestion d'urgence pour lesquelles il n'est pas systématiquement possible d'interrompre son activité ou celles des autres pour donner de l'information (Pavard, Benchekroun, \& Salembier, 1990 ; Heath, \& Luff, 1992 ; Filippi, \& Theureau, 1995). En effet malgré la nécessité pour chacun de rester informé des activités en cours ou projetées des autres agents il n'est pas toujours possible ni même souhaitable de délivrer cette information à tout prix : en période de forte sollicitation l'opérateur ne va pas forcément pouvoir ou vouloir interrompre son activité pour informer ses collègues. De la même façon cette information n'a pas vocation à être systématiquement transmise.

L'accès conjoint aux ressources, pour important qu'il soit, n'est néanmoins pas suffisant pour garantir les conditions d'une coordination des activités individuelles et d'une coopération entre agents efficaces. Il ne suffit en effet pas de rassembler en un même lieu des opérateurs formés travaillant sur le même procès pour obtenir un fonctionnement satisfaisant du collectif. D'autres facteurs entrent en ligne de compte, au nombre desquels on pourra citer :

- La sensibilité à l'accomplissement en temps réel de l'activité en train de se faire des autres acteurs constitue une condition pour la coordination efficace des actions du collectif, et partant, pour la réalisation satisfaisante des tâches qui lui sont assignées (Heath, \& Luff, 2000).

- La capacité des agents à interpréter correctement les événements de la situation (les actions des tiers, les traces de ces actions, les arrangements spatiaux des objets, ...) requiert une connaissance fine de la situation et de ses pratiques locales historiquement et culturellement constituées (Rognin, Salembier, \& Zouinar, 1998 ; Salembier, 2002). Ces événements doivent en effet être signifiants pour l'acteur sinon on a affaire à une simple réactivité aléatoire.

- Le niveau de disponibilité des agents joue également un rôle important. Une grande variété d'indices est susceptible de renseigner sur la disponibilité de chacun des agents et donc sur la possibilité de les utiliser comme ressource éventuelle. Cet aspect a été finement documenté dans l'étude mené par Pavard et ses collègues dans les salles de régulation des appels d'urgence du SAMU et repris dans le domaine du contrôle aérien.

- La capacité à déterminer quelles sont les informations utiles à transmettre, à quel moment et sous quelle forme elles doivent être diffusées, et si les autres agents sont en mesure de les recevoir compte tenu par exemple de leur niveau de disponibilité (Benchekroun, Pavard, Medeiros, \& Salembier, 1990 ; Goodwin, \& Goodwin, 1996) requièrent également une forme de compétence particulière. 
- La labilité relative des supports de représentation peut faciliter ou alourdir les éventualités d'accès conjoint aux ressources : ainsi la permanence physique d'une information sera moindre si elle est énoncée à voix haute que si elle est écrite sur un papier, sur un écran ou inscrite durablement dans une configuration spatiale d'objets ${ }^{1}$.

\section{3.- L' «Accountability»: sésame conceptuel de l'approche éthnométhodologique}

Comment théoriser la question de la construction de l'intelligibilité mutuelle dans l'activité collective ? Le concept d'"accountability" constitue une voie de réflexion difficilement contournable.

L"'accountability", que l'on traduira classiquement, bien que sans doute imparfaitement, par descriptibilité, et plus largement l'ensemble de l'oeuvre de Garfinkel, a donné lieu à une somme exégétique et critique considérable [voir par exemple (Heritage, 1984, 1987 ; Livingstone, 1987 ; Fornel, Ogien, \& Quéré, 2001)] souvent destinée à un public de spécialistes. Notre propos n'est pas ici d'intervenir dans ces discussions mais d'examiner :

- en quoi la "descriptibilité » publique, qui sous-tend toute l'approche de l'analyse de l'action développée par Garfinkel, offre-t-elle une voie de thématisation aux questions posées par l'étude de l'accès conjoint aux ressources dans le contexte d'activités collectives requiérant des formes diverses de coordination entre les acteurs ;

- quelle utilisation a été faite de ce concept dans la perspective plus technologique d'une contribution à la conception de l'interaction homme-machine et de l'interaction homme-homme médiée par des artefacts.

\section{1.- Bref rappel sur la notion de «descriptibilité »}

Une dimension spécifique de l'approche de Garfinkel pour rendre compte de l'ordre social réside dans le fait que pour lui, les acteurs organisent leurs actions et leurs interactions de façon à les rendre restituables, sujettes à description et donc mutuellement intelligibles. Selon sa formulation initiale,

« Les études ethnométhodologiques analysent les activités quotidiennes en tant que méthodes mises en œuvre par les membres ${ }^{2}$ pour rendre ces dites activités visiblement rationnelles et rapportables à toutes fins pratiques, i.e. "descriptibles" en tant qu'organisations des activités quotidiennes ordinaires ». (Garfinkel, 1967)

Un des aspects clés de la pensée de Garfinkel réside dans le fait que les moyens par lesquels la séquentialité de l'activité est organisée sont les mêmes que ceux par lesquels elle est reconnue : ce qui signifie que les ressources pour comprendre une activité donnée sont inscrits dans l'organisation séquentielle de cette activité. C'est ce à quoi réfère le terme de « descriptibilité » de l'action.

Garfinkel s'intéresse donc à la manière dont les actions donnent lieu à des représentations publiques qui sont accessibles aux autres acteurs. Dans ce cadre, la « descriptibilité » a trait aux méthodes uti-

1. On retrouve ici, rabattue sur une problématique de coordination, la question des rapports entre mémoire et technologie, et plus généralement de l'extériorisation matérielle de l'objet de pensée. Au-delà du traitement empirique évoqué dans cet article, cette question fait l'objet d'une réflexion philosophique considérable. Elle est notamment au coeur du travail mené par Bernard Stiegler depuis plusieurs années (Stiegler, 1996).

2. Par « membre » Garfinkel entend membre d'une communauté ou d'un groupe 
lisées par une communauté d'agents pour rendre leur activité intelligible dans un contexte social donné et au rôle de l'interaction dans ce processus de mise en visibilité de l'action pour les autres.

On peut également envisager une autre façon d'entendre la descriptibilité qui interroge la possibilité pour un agent d'adopter une posture réflexive sur son activité afin d'en produire une description à un tiers plus ou moins informé, par exemple un autre acteur afin de lever un malentendu ou une ambiguité lors d'une interaction, mais également par extension un analyste qu'il soit ergonome ou ethnométhodologue (voir l'article de Theureau dans ce numéro). Cette acception est d'une certaine façon plus proche de ce qu'Heritage a défini comme «le second niveau de la descriptibilité » :

«Par ailleurs, il y a le niveau de l'explication manifeste par laquelle les acteurs sociaux donne une explication de ce qu'ils sont en train de faire en termes de raisons, mobiles ou causes ». (Heritage, 1990) p. 26.

Il convient néanmoins de ne pas perdre de vue que pour Garfinkel c'est l'ensemble des méthodes de raisonnement tacites, socialement organisées et partagées par les acteurs qui rendent leurs comportements intelligibles et que les explications qu'ils peuvent éventuellement produire à propos de leurs actions doivent être articulées avec cet arrière-plan de compréhensions implicites déja établies :

«Comme le souligne Garfinkel : « les activités par lesquelles les membres produisent et gèrent les cadres des activités ordinaires organisées sont identiques aux procédures mises en œuvre par ces membres pour rendre ces cadres "descriptibles" (accountable)». Cette citation pourrait être entendue comme impliquant que c'est principalement lorsque les membres produisent des explications de leur conduite que leur comportement devient intelligible. Mais c'est l'inverse de la position de Garfinkel. En réalité, pour lui, les bases méthodologiques ou procédurales du raisonnement génèrent de manière continue des compréhensions implicites actualisées de ce qui est train de se produire dans l'interaction sociale - un «index courant» (running index), ainsi nommé, de ce qui survient dans un événement social. C'est à travers la création de cet «index courant » que l'activité sociale est rendue intelligible ou, comme énoncé par Garfinkel « descriptible ». (Ibid., p. 26)

\section{2.- Les enrôlements de la « descriptibilité » dans la conception}

Un rapide examen de la littérature montre que la reférence à la notion de descriptibilité est omniprésente dans les travaux ethnométhodologiques orientés vers la conception de systèmes interactifs et plus particulièrement de systèmes d'aides à la coopération $(\mathrm{CSCW})^{1}$. Ce lien entre descriptibilité et conception a été envisagé essentiellement sous deux angles :

\section{Dans le domaine de l'interaction Homme-machine}

L'idée est ici de donner à l'utilisateur un certain accès dynamique à ce que fait le système dans des termes qui soient pertinents, i.e. qui établissent une relation entre l'état du système et l'état du travail dans lequel est engagé l'utilisateur, de façon à ce que celui-ci puisse organiser de manière compréhensive son activité courante (Dourish, 1995). L'articulation avec l"'" action située "” réside dans le fait que le système fournit de manière continue des représentations computationnelles de ses activités comme ressources pour la conduite de l'action improvisée et contextualisée. Un des aspects innovants de la démarche, qui sinon ne se démarquerait des travaux classiques en interaction homme-machine que par l'exotisme quelque peu artificiel de sa source d'inspiration, réside dans le fait que la représentation fournie par le système de son activité est construite sur la base de descriptions (accounts) générés par les capacités de reflexivité du système et non pas à partir d'un modèle extérieur, intégré par conception.

1. Pour une discussion de la relation entre « descriptibilité » et technologie (cf. Suchman,1993). 
A partir de cette tentative d'application des travaux ethnométhodologiques, Dourish a forgé le concept de « descriptibilité computationnelle » (computational accountability) (Dourish, 1996 ; Button $\&$ Dourish, 1996) qui selon nous constitue essentiellement une déclinaison « comportementale $»^{1} \mathrm{de}$ la notion de scrutabilité des systèmes ${ }^{2}$. De ce point de vue, et comme le signale à juste titre Eriksen (Eriksen, 2002), la « descriptibilité computationnelle» reste finalement assez proche de la notion de « transparence » (Agnér Sigbo, 1993) reprise et actualisée par exemple dans le domaine des systèmes d'interaction en langage naturel (Karsenty, 2002).

\section{Dans le domaine de l'interaction Homme-Homme médiatisé par des artefacts informatiques.}

On s'intéresse ici à des situations où les agents, qui ne sont pas en situation d'interaction en face-àface voire ne sont pas co-présents dans le même espace, ont recours à des outils de communication et de représentation (media-spaces, vidéo-conferences, ...) qui permettent une forme de présentification matérielle de l'interlocuteur absent, et l'émergence d'une «conscience mutuelle » ${ }^{3}$ (mutual awareness) entre agents coopérants. C'est en fait une grande part de la problématique technologique du courant $\mathrm{CSCW}^{4}$, qui vise à assurer les conditions matérielles de ce que nous avons appelé « une assistance par la régulation émergente » (Salembier et al., 2001).

Le terme d'awareness est généralement utilisé en reférence à l'ensemble des pratiques qui permettent l'ajustement et l'intégration tacites et non intrusifs des activités coopératives. Cette « conscience » mobilise un double processus bouclé : la surveillance plus ou moins diffuse des événements qui se produisent dans la situation, et notamment l'activité des autres opérateurs, et dans le même temps la mise en visibilité des aspects de sa propre activité qui peuvent être pertinents pour les autres (voir l'exemple détaillé plus haut).

«Rendre visible et surveiller sont par conséquent des aspects complémentaires des mêmes pratiques de coordination. La surveillance que j'effectue de l'activité des autres est facilitée par le fait qu'ils donnent à voir ces aspects qui sont pertinents pour moi, et le fait que je rende visible certains aspects de mon travail aux autres présuppose que je suis attentif à leurs activités, et par là même au courant de leurs préoccupations, de leurs attentes et de leurs intentions. » (Schmidt, 2002a)

Dans le contexte du CSCW, une première approche de la restitution des propriétés de « descriptibilité » inhérentes aux situations de co-présence physique a consisté à généraliser l'usage de systèmes informatiques intégrant audio et vidéo. Le modèle appliqué classiquement ici est de prendre comme situation de reférence la communication en face-à-face, et de trouver la solution technique qui s'en approche le plus. L'échec relatif de ces systèmes ${ }^{5}$ (dont le media-space est l'exemple le plus connu) a conduit à envisager d'autres alternatives, comme l'utilisation de milieux virtuels ou la dissémination automatique d'informations jugées utiles pour la coopération sur des réseaux. Dans certaines situations où les opérateurs sont co-présents physiquement mais où l'aménagement de l'espace et/ou les exigences de l'activité confinent les opérateurs sur leur poste de tra-

1. «Comportementale » car le système donne ici une représentation de son état et des traitements en cours et non pas une représentation de sa compétence fonctionnelle (compréhension d'une situation problème à résoudre, d'un cas soumis pour traitement, ...) ou interactionnelle (compréhension d'une question posée par l'utilisateur).

2. Point abondamment traitée dans les travaux sur les modèles informatiques de l'expertise, voir les discussions sur les modèles de type « boîte-noire » et « boîte-de-verre » (Anderson, 1988).

3. « Conscience » est une traduction peu satisfaisante du terme anglais awareness qui recouvre lui-même, dans le contexte qui nous occupe, des significations extrêmement variées et parfois contradictoires (Schmidt, 2002a)

4. L'autre grand courant de recherche dans le $\mathrm{CSCW}$ a pour objectif de fournir des supports à la coordination et à l'interaction au travers de modèles normatifs de la coopération. Les outils de type workflow sont représentatifs de cette approche que nous avons ailleurs qualifiée « d'assistance par la régulation prescriptive ».

5. Voir par exemple (Cardon, 1997) pour une bonne analyse critique de cet échec. 
vail, des collecticiels ont été développés qui permettent d'entreméler modes naturels d'accès à l'activité des tiers (par l'exemple par l'écoute flottante) et modes assistés par le système informatique (voir notamment le projet exemplaire menée par Pavard et ses collègues dans les salles de régulation des appels d'urgence (Pougès, Guy, Pavard, Gourbeault, \& Champion, 1994).

\section{4.- Quelle approche du contexte partagé pour quelle finalité?}

\section{1.- De l'Accountability de l'action aux représentations communes}

L'ensemble des études cités plus haut mettent en évidence, sous des formes diverses, le rôle fondamental joué par l'accès conjoint aux informations contextuelles dans le fonctionnement des collectifs de travail. Il facilite la mise en œuvre de processus d'interaction efficaces entre agents, la coordination des actions, la résolution en commun de problèmes et la mise en place contingente ou réglée d épisodes d'aide mutuelle. Le partage d'informations joue ainsi un rôle crucial dans le fonctionnement efficace et fiable d'une catégorie de situations que Suchman a défini comme « centres de coordination »; cette catégorie couvre notamment les situation de contrôle et de supervision de processus dynamiques, souvent associées à un haut niveau de risque humain et matériel (McCarthy, Wright, Healey, \& Harrison, 1997 ; Rognin, Salembier, \& Zouinar, 1997).

A côté de l'approche d'inspiration éthnométhodologique évoquée plus haut ${ }^{1}$, mais sur la base de positions radicalement différentes, d'autres champs disciplinaires se sont attachés à traiter dans une optique représentationnaliste la question de la circulation, de l'accès, et du partage d'informations comme condition ou ressource pour l'organisation de l'activité collective. La majorité de ces approches mobilisent des cadres théoriques différents et des notions spécifiques (voir par exemple Zouinar, 2000). Elles partagent néanmoins une reférence à l'existence préalable de connaissances et de représentations plus ou moins stabilisées qui constituent la condition de réalisation de l'action collective, et ce à la différence de l'ethnométhodologie pour laquelle ces éléments constituent des productions émergentes de l'accomplissement de l'action et de l'accès conjoint aux ressources.

La notion de savoir (de connaissances ou de croyances) mutuel (ou commun ou encore partagé) est sans doute la plus courante dans la littérature; elle a été essentiellement thématisée dans le champ de la philosophie du langage et la plupart des autres notions s'y réfèrent d'ailleurs plus ou moins explicitement et à des degrés divers. Le point qui sous-tend ces différentes approches est que partager de l'information c'est au bout du compte posséder des connaissances, des croyances, des savoirs identiques ou compatibles quant à leurs contenus. Ce qui est partagé peut concerner des éléments de l'ici et maintenant d'une situation (action d'un agent, événement, état à un moment donné d'un objet, etc.) ou des éléments que l'on pourrait qualifier « d'arrière-plan » (par exemple, connaissances ou croyances locales de métier historiquement et culturellement constituées et connaissances ou croyances plus "générales » inscrites dans un champ plus large que le contexte professionnel par exemple).

On retrouve ce positionnement général dans les travaux menés en ergonomie et en psychologie du travail sur la construction d'une représentation fonctionnelle commune de la tâche à fins de coordination des activités individuelles. Cette représentation est ici conçue comme « la partie de la représentation de chacun qui est commune avec celle des autres membres du groupe » (Leplat, 1997). Ainsi, la notion de « conscience de la situation de groupe » (Team Situation Awareness), qui est une

1. A laquelle on pourra ajouter la sociologie phénoménologique de Schütz qui ne sera pas abordée dans cette article mais qui occupe une place considérable dans la compréhension du fonctionnement des collectifs (voir par exemple : Grosjean, \& Lacoste, 1999 ; Cefaï, \& Depraz, 2001 ; Schmidt, 2002b) et dont on peut considérer qu'elle constitue également une source d'inspiration importante de l'«action située». 
extension collective de la notion de « conscience de la situation » (situation awareness) (Endsley, 1995) emblématique des recherches menées dans l'aéronautique depuis plusieurs années, renvoie au partage de connaissances sur des éléments pertinents de la situation (Salas, Prince, Baker, \& Shrestha, 1995). La notion de « référentiel opératif commun » se réfère à une représentation qui est commune aux agents qui ont à réaliser un même objectif donné (Terssac, \& Chabaud, 1990). Cette représentation est opérative et transitoire, dans la mesure où elle sert à la préparation et à la réalisation de l'action visée par les opérateurs, et se constitue à l'occasion d'une tâche particulière. Elle est élaborée conjointement par une mise en commun des compétences de chacun et renvoie à une mise en commun de savoirs et de savoir-faire nécessaires à la réalisation d'un travail donné.

Le partage peut prendre différentes formes. Dans sa formulation «simple» il n'implique pas un emboîtement infini de connaissances ou de croyances (par exemple, A sait que $\mathrm{p}^{1}$, B sait que $\mathrm{p}, \mathrm{A}$ sait (ou croit) que $B$ sait que $p, B$ sait que $A$ sait que $p, \ldots$ ad libidum) dans la mesure où il définit plus ou moins arbitrairement un point d'arrêt de la séquence. Dans sa forme « mutuelle » il implique logiquement cet emboîtement. Cette définition pose alors un problème classique de régression à l'infini de connaissances emboîtées (Livet, 1994 ; Sperber, \& Wilson, 1995) qui obère sa plausibilité psychologique, dans la mesure où la réflexivité qu'elle implique suppose des ressources cognitives qui dépassent largement celles dont dispose un agent humain. Dans le cadre du CSCW Schmidt a proposé une réponse pragmatique à ce problème en distinguant clairement «mutuel» de « réciproque » (Schmidt, 1998) et en relaxant les contraintes logiques de récursivité habituellement associées à la notion de mutualité. Nous proposerons plus loin une approche du partage «mutuel » d'informations qui permet de contourner cette difficulté d'une autre façon (voir également (Zouinar, 2000).

\section{2.- Le problème de l'efficacité opératoire des notions dans un programme de recherche technologique}

Un problème important commun à l'ensemble des approches représentationnalistes et logiques que nous avons évoquées est qu'elles répondent de façon peu satisfaisante aux exigences du versant technologique de notre programme à savoir l'évaluation des conséquences de la (re)conception de futures situations sur le partage d'informations contextuelles en recourant à une forme de "simulation cognitive" 2 , qui présente l'avantage de permettre l'évaluation de plusieurs alternatives de conception à des coûts plus faibles.

Ces approches sont en effet basées sur des notions qui ne permettent pas de traiter la question du non déterminisme du partage d'information : il n'est pas possible de prévoir avec certitude les informations qui seront ou non partagées par les agents dans des situations qui n'existent pas encore, car nous ne pouvons tout simplement pas délimiter tous les facteurs susceptibles de conditionner les activités futures, surtout dans des situations complexes telles que le trafic aérien. Si l'on tente d'appliquer des notions déterministes on ne peut choisir qu'entre deux options radicalement opposées : soit l'information est partagée, soit elle ne l'est pas. Or, de notre point de vue, le fait de dire qu'une information est partagée doit être considéré comme une possibilité ou une hypothèse : l'hypothèse la plus forte sur un axe dont l'autre point extrême désigne une forte probabilité que

1. Dans cet exemple, $\mathrm{A} \& \mathrm{~B}$ sont deux agents, et « $\mathrm{p}$ » représente une proposition qui se réfère à une chose, un événement ou à un état de choses.

2. Telle que nous l'entendons ici, la simulation cognitive est à regarder comme une projection virtuelle systématisée d'une situation d'activité future, moyennant un certain nombre d'hypothèses sur cette situation. Elle constitue d'une certaine façon, dans le contexte de situations proches de celles que nous étudions, le pendant ergonomique, c'est-à-dire technologique, de simulations qui se donnent pour objectif de reproduire de manière psychologiquement plausible toute ou partie d'une activité cognitive donnée (Woods, Roth, \& Pople, 1987 ; Cacciabue, Decortis, Drozdowicz, Masson, \& Nordvik, 1990 ; Amalberti, 1992). 
l'information ne soit pas partagée. Les hypothèses sur lesquelles reposent les approches en termes de connaissance ou croyance mutuelles sont beaucoup plus fortes car elles sont essentiellement binaires et régies par un principe de vériconditionnalité stricte.

En outre, ces notions ne permettent pas de prendre en compte le fait que dans ces situations, les informations auront plus ou moins de chance d'être partagées, en fonction des circonstances. Tenir compte de ce fait est important, car cela ouvre des possibilités d'évaluations plus fines de l'impact des modifications des environnements de travail sur le partage d'informations. Par exemple, on peut se demander dans quel(s) environnement(s) une information aura le plus de chance d'être partagée par les agents concernés. En d'autres termes, cela permet de disposer d'un «critère » permettant d'aider à choisir entre différentes options de conception, du point de vue du partage d'informations.

Pour tenter de dépasser ces limites des approches « binaires », nous avons développé une approche du partage d'informations contextuelles qui s'appuie sur la notion de manifesteté mutuelle telle que spécifiée par Sperber et Wilson (Op. Cit.).

\section{5.- De la « Manifesteté Mutuelle » au «Contexte Partagé »}

\section{1.- La notion de Manifesteté mutuelle}

Pour expliquer le fonctionnement de la communication et la manière dont des agents arrivent à se comprendre, des philosophes et des psychologues ont développé une théorie fondée sur la notion de connaissance ou savoir mutuel. Par exemple, supposons que suite à un premier échange entre deux agents $\mathrm{A}$ et $\mathrm{B}$, $\mathrm{A}$ fasse la remarque suivante : « il n'aurait jamais dû partir » en pensant à un agent $\mathrm{C}$, ami de $\mathrm{A}$ et $\mathrm{B}$ dont ils sont en train de parler. Pour que l'énoncé soit compris, A doit alors attribuer à $\mathrm{B}$ la connaissance que la personne à qui il fait référence est $\mathrm{C}$, que $\mathrm{B}$ sache que $\mathrm{A}$ sait que $\mathrm{B}$ sait que la personne en question est $\mathrm{C}$, que $\mathrm{A}$ sache que $\mathrm{B}$ sait que $\mathrm{A}$ sait que la personne référée est $\mathrm{C}$, et ainsi de suite à l'infini. Comme précisé plus haut, la théorie de la connaissance mutuelle a donc pour caractéristique de produire une régression à l'infini. Cette théorie ne peut donc être intégrée à une explication cognitivement adéquate de la production et de la compréhension des actes communicatifs, car comme nous l'avons déjà souligné, elle suppose des capacités de raisonnement inférentielles phénoménales hors de portée d'un agent humain lambda. Ce qui a pour conséquence que l'hypothèse du savoir mutuel ne peut jamais être validée, dans la mesure où ce savoir ne peut se réaliser ou être atteint par les agents.

Partant de ces critiques et admettant que toute analyse de la communication humaine ne peut faire l'économie d'une notion d'information partagée, Sperber et Wilson ont développé une notion plus « faible » mais empiriquement plus adéquate que celle de connaissance mutuelle. Cette notion, la «manifesteté mutuelle », repose sur les concepts de manifesteté et d'environnement cognitif.

\subsection{1.- Manifesteté et Environnement Cognitif}

Pour Sperber et Wilson, un fait est manifeste pour un individu s'il est capable d'en construire une représentation et d'accepter cette représentation comme vraie ou probablement vraie.

«Un fait est manifeste à un individu à un moment donné si et seulement si cet individu est capable à ce moment-là de représenter mentalement ce fait et d'accepter sa représentation comme étant vraie ou probablement vraie. » (Sperber, \& Wilson, op. cit., p. 65)

En d'autres termes, un fait manifeste est un fait qui a la caractéristique d'être perceptible ou inférable par un agent à un instant donné. Dans cette perspective, un fait peut être manifeste sans être effectivement connu. Ainsi, dire qu'un fait est manifeste, c'est lui attribuer une propriété plus faible que celle qu'on lui attribuerait en disant qu'il est connu ou même supposé. En outre, certains faits peu- 
vent être plus manifestes que d'autres. Il y a des faits qui ont plus de chance d'être perçus ou inférés que d'autres. A chaque fait, on peut donc associer un degré de manifesteté qui est fonction des capacités perceptuelles et cognitives de l'individu, et de son environnement physique. Par exemple, supposons qu'un téléphone sonne dans une pièce où se trouve un individu $\mathrm{A}$, et qu'au même moment une voiture passe dans une rue sur laquelle donne la pièce. Dans ce cas, toutes choses égales par ailleurs, il sera fortement manifeste pour A que le téléphone sonne, mais moins manifeste qu'une voiture est passée. Il convient de noter que ceci ne veut pas dire que ce dernier fait ne sera pas perçu, mais simplement qu'il aura moins de chance de l'être car la sonnerie du téléphone est, étant donné la présence de A dans la pièce, perceptuellement plus «saillante ». Ainsi, du fait de la différence de saillance entre la sonnerie du téléphone et le bruit de la voiture, le fait « le téléphone sonne » est plus manifeste, c'est-à-dire a plus de chance d'être perçu ou inféré que le fait « une voiture est passée ».

Partant de cette notion de manifesteté, Sperber et Wilson définissent celle d'environnement cognitif, qui représente l'ensemble de tous les faits qui sont manifestes pour un individu donné, c'est-à-dire tous les faits qu'il peut percevoir ou inférer. L'environnement cognitif total d'un individu désigne tous les faits qui lui sont manifestes, y compris ceux dont il a pris connaissance.

\subsection{2.- Environnement Cognitif Partagé et Environnement Cognitif Mutuel}

Un Environnement Cognitif Partagé (ECP) désigne une intersection d'environnements cognitifs individuels. Il désigne tous les faits qui sont manifestes à deux ou plusieurs individus. Reprenons l'exemple du téléphone en imaginant qu'un autre individu B se trouve dans la même pièce que A. Dans ce cas, en supposant qu'ils aient les mêmes capacités perceptuelles, il est alors manifeste pour A et pour B que le téléphone sonne. Ceci signifie simplement qu'ils sont capables de percevoir ou d'inférer le même fait, et non qu'ils partagent réellement une croyance, une connaissance, ou une représentation concernant ce fait.

L'Environnement Cognitif Mutuel (ECM) désigne un ECP dans lequel l'identité des individus qui ont accès à cet environnement est manifeste. Si l'on reprend l'exemple ci-dessus, A et B partagent un environnement cognitif qui comprend tous les faits qui leur sont rendus manifestes par leur co-présence dans la même pièce. L'un de ces faits est qu'ils partagent cet environnement.

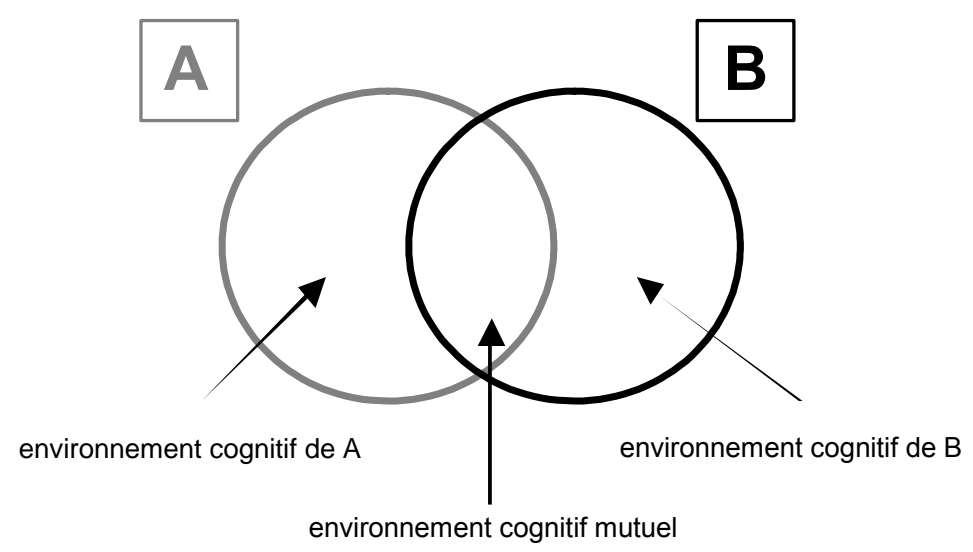

Figure 3.- Représentation graphique de la relation entre environnements cognitifs individuels et ECM (les lettres A \& B représentent deux agents).

Dans l'ECM, tout fait manifeste est dit mutuellement manifeste. Ainsi, dans l'exemple du téléphone, on peut distinguer un nombre illimité de faits mutuellement manifestes pour A et pour B : le télé- 
phone sonne, il est manifeste pour $\mathrm{A}$ et pour B que le téléphone sonne, il est manifeste pour A et pour B qu'il est manifeste pour A et pour B que le téléphone sonne, etc. Plus ces faits sont complexes moins ils ont de chance d'être perçus ou inférés par les deux agents ; autrement dit, ils ont un degré de manifesteté mutuelle, qui correspond donc à une sorte de « probabilité » qu'ils soient perçus ou inférés par les agents considérés. La manifesteté mutuelle est ainsi une notion conceptuellement beaucoup plus faible que celle de connaissance, de croyance, de représentation mentale partagées, car elle porte sur des faits potentiellement perceptibles ou inférables, et non pas sur d'hypothétiques états cognitifs effectifs ou réalisés.

\section{2.- Contexte partagé}

Du fait qu'elle permet de distinguer différents degrés de partage de l'information, la manifesteté mutuelle apparaît comme une notion adéquate pour traiter les incertitudes liées à l'analyse du partage d'informations. En outre, elle constitue un moyen utile et pertinent pour analyser ce phénomène dans le cadre d'analyse du partage d'informations, dans des environnements projetés. Cependant, du point de vue de l'étude d'activités finalisées dans des environnements professionnels donnés, elle n'est pas exploitable telle qu'elle ; elle ne fait en effet pas de différence entre ce qui peut être pertinent pour les acteurs/opérateurs et ce qui ne l'est pas, relativement aux tâches qu'ils doivent réaliser et à leurs activités du moment.

Ce point nous a conduit à opérer une délimitation au sein de ce qui constitue l'environnement cognitif mutuel (figure 4). Ainsi, nous avons nommé contexte partagé un ensemble d'informations ou événements contextuels mutuellement manifestes pour un ensemble d'acteurs, à un instant $t$ dans une situation donnée, compte tenu de leurs capacités perceptuelles et cognitives, des tâches qu'ils doivent réaliser, et de leur activité en cours. Par "événements contextuels», nous entendons l'ensemble des événements qui se manifestent au cours d'une activité et qui sont ou peuvent être pertinents relativement à la réalisation de cette activité et ceci du point de vue des acteurs. Ces événements sont qualifiés de contextuels car ils sont relatifs à un contexte d'activité particulier.

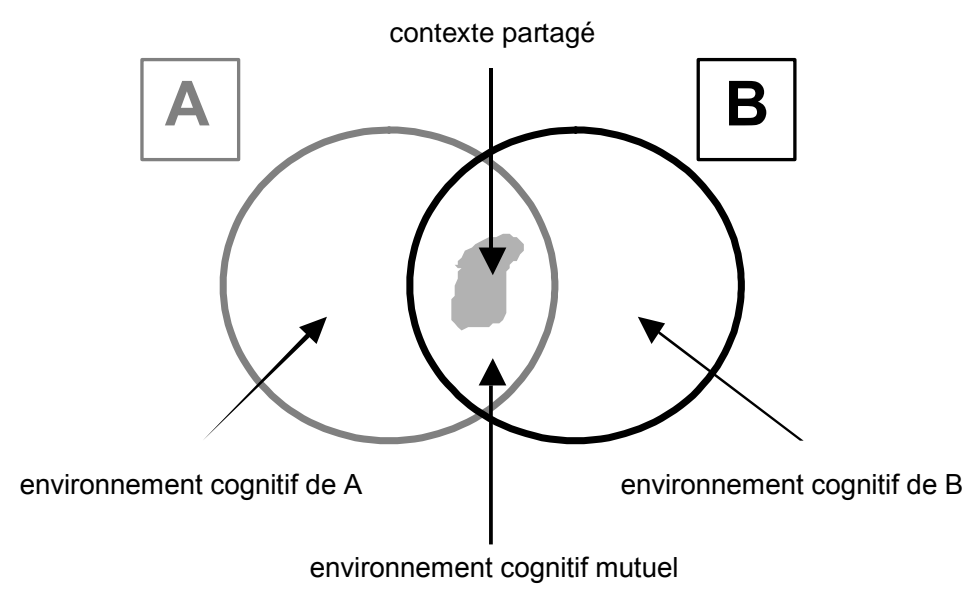

Figure 4.- Représentation graphique de la relation entre ECM et Contexte Partagé (les lettres A \& B représentent deux agents).

Cette notion de contexte partagé appelle quelques précisions. Il est clair que dans une situation de travail les opérateurs ne sont pas des spectateurs inertes, non concernés par ce qui se passe autour d'eux. Les événements, et notamment les actions des tiers, qui se déroulent sont signifiants pour eux 
sans qu'ils aient la plupart du temps à faire un effort particulier pour leur donner du sens (Schmidt, 2002a). Tout le problème est donc de parvenir à identifier, parmi les éléments manifestes de la situation ceux qui sont susceptibles d'étre considérés par les opérateurs. Cette identification peut être réalisée sur la base de la connaissance du domaine par l'analyste : un bon niveau de familiarité avec la situation (qui peut aller jusqu'à une pratique personnelle confirmée de l'activité considérée ${ }^{1}$ ), permet dans une certaine mesure d'opérer une réduction valide de l'espace des événements pertinents. Sous cet angle le contexte partagé est donc une construction théorique qui modélise de manière extrinsèque un phénomène intuitif particulier qui est que les êtres humains partagent ou peuvent partager de l'information : cette construction est en effet le fait d'un observateur extérieur qui opère de facto un filtrage des éléments supposés signifiants pour les agents. Mais, dans la démarche que nous avons mise en place (Salembier, Kahn, Zorola-Villarreal, \& Zouinar, 1997 ; Zouinar, \& Salembier, 2000), ce filtrage est guidé par une connaissance de l'activité acquise grâce à de nombreuses heures d'observation, d'analyse et d'entretiens avec les opérateurs et des experts du domaine.

\section{3.- Mise en oeuvre du programme technologique : contexte partagé et simulation}

La reformulation que nous avons donnée du problème de l'accès conjoint aux informations contextuelles et des représentations partagées ou mutuelles constitue un entre-deux entre un externalisme extrême et une conception représentationnaliste du partage d'informations. Cette approche permet d'une part de répondre à la question du non-déterminisme et par ailleurs convient bien à la perspective de projet technologique que nous menons parallèlement à réflexion sur l'objet théorique « contexte partagé ». Elle permet en effet une transition entre les analyses empiriques extensives menées en situation, la modélisation basée sur un processus de littéralisation reposant sur un formalisme logico-mathématique, et la simulation informatique qui constitue la dernière phase de la démarche (Salembier, \& Pavard, 2004).

Cette simulation (dont nous ne donnerons pas les détails ici ; voir Zouinar, Op. cit, pour une présentation complète) utilise une plateforme informatique dédiée qui permet de générer automatiquement des données pour l'évaluation de différents scénarii de (re)conception de situations de travail, sur le critères du volume et de la nature d'événements mutuellement manifestes signifiants pour les opérateurs. Ces sorties de simulation sont ensuite analysées et peuvent constituer la base d'échanges avec les concepteurs et les opérateurs (Salembier et al., 1997).

Il convient de noter que la réalisation de ce programme conduit dans l'état actuel de nos travaux à une inévitable réduction technologique de l'objet théorique initial. Cette réduction ne porte pas sur la nature de l'objet même, mais sur les modalités de sa description et de sa formalisation ainsi que sur le dimensionnement des phénomènes considérés : ce qui est traité ici ce ne sont pas les mécanismes cognitifs de génération et d'actualisation du contexte partagé, mais les conditions de leur mise oeuvre et les conséquences, évaluées quantitativement, de leurs modifications. Par ailleurs les impératifs de la simulation conduisent à utiliser un langage de formalisation qui implique certaines opérations (discrétisation du processus, énumération comptable d'états, ...) qui peut donner l'impression d'une conception statique et déterministe du contexte partagé alors que pour nous il s'agit bien d'un processus inséparable de la dynamique de l'activité, et qui va donc se construire et évoluer en fonction des contingences de la situation et des actions des agents.

1. Dans cette catégorie on pourra citer l'exemple d'Hutchins dont les études sur les navigateurs micronésiens et sur les pilote de ligne sont bien connues, et qui est lui-même un navigateur confirmé et qui pilote régulièrement. 


\section{6.- Conclusion}

Nous avons choisi dans cet article d'aborder un aspect précis de l'« action située » à savoir l'accent mis sur les processus de restitution publique de l'activité de chacun des agents à leur collègues et leur rôle sur la production d'intelligibilité mutuelle en temps réel. Cette question a fait l'objet de nombreux travaux, dans des cadres disciplinaires variés, notamment en ethnométhodologie et en psychologie ergonomique. Elle offre l'intérêt d'un ancrage théorique fort (autour des notions de « descriptibilité » et de " contexte partagé » notamment) et d'un volume d'analyses empiriques conséquent, menées dans des situations variées, souvent articulées avec des préoccupations de conception non triviales.

Dans le cas de l'ethnométhodologie ces analyses, aussi fines et informatives soient-elles, ouvrent sur une critique possible : l'attention est ici essentiellement centrée sur l'identification et la description des processus de mise en visibilité, sans s'intéresser nécessairement au contenu, c'est-à-dire à la signification pour l'acteur de ce qui est rendu visible, ou plus exactement de la signification pour l'acteur telle qu'on peut y avoir accès a posteriori dans un discours retrospectif. Pour l'ethnométhodologie ce qui prime c'est la notion phénoménologique de signification in vivo ${ }^{1}$. Il y a d'ailleurs de ce point de vue une certaine cohérence entre la définition de l'objet d'étude et la méthode d'appréhension de cet objet (voir l'introduction à ce numéro). On pourrait avancer que, dans le cadre de cohérence ontologique et épistémologique défini par Garfinkel et l'ethnométhodologie, cette critique ne fait pas problème dans la mesure où la signification qu'un acteur donne à son activité, telle qu'il peut la restituer à un analyste extérieur, n'est pas l'objet théorique de l'ethnométhodologie. Il reste que cette position prive volontairement l'analyse d'un ensemble de données riches susceptibles d'éclairer l'activité. Dans la perspective d'une confrontation multi-disciplinaire autour du thème de l'organisation et de la signification des activités cette question prend donc tout son sens.

D'une certaine façon l'approche que nous avons proposée n'échappe pas à ce type de critique : le fait qu'un événement soit mutuellement manifeste indique que les agents concernés peuvent potentiellement en avoir mutuellement conscience mais il ne donne pas d'éléments sur la signification de cet événement pour les agents ni sur les défauts éventuels d'intelligibilité mutuelle (un même événement peut être compris, interprété différemment, voire même parfois de façon contradictoire; seule une analyse fine permettra alors d'en donner une explication). Le problème de la signification est partiellement considéré en amont lors de la phase d'analyse fine des activités qui permet d'identifier les classes d'événements pertinents grâce à l'explicitation qui en est donnée par les acteurs à partir d'enregistrements de leur activité. Mais ceci reste néanmoins insuffisant, même dans une perspective uniquement technologique de (re) conception des situations; les simulations informatiques effectuées ne constituent en effet qu'une étape dont les résultats doivent être complétés par des approches plus classiques d'évaluation.

\section{BIBLIOGRAPHIE}

Agnér Sigbo, G. (1993). Seeing through computer systems. The importance of models for use an development of computer systems. In L. Lennerlöf (Ed.), People, Computer Technology, Working Life. Stockholm: Publica.

Agre, P. E. (1993). The symbolic worldview : reply to Vera and Simon. Cognitive Science, 17 (1), 7-48.

Amalberti, R. (1992). Modèles d'activités en conduite de processus rapides : implications pour l'assistance à la conduite. Unpublished Thèse de doctorat, Université Paris VIII.

Anderson, J. R. (1988). The expert module. In M. C. Polson, \& J. J. Richardson (Eds.), Foundations of Intelligent Tutoring Systems (pp. 21-54). Hillsdale, NJ: LEA.

1. Merci à Marc Relieu d'avoir attiré notre attention sur ce point 
Benchekroun, T. H., Pavard, B., Medeiros, E. d., \& Salembier, P. (1990). Analyse et modélisation des interactions dans un centre de régulation des appels d'urgence. Paper presented at the XXVIe congrès de la SELF.

Bressolle, M. C. (1993). Contribution à l'étude de la perception de l'intention et de la coopération. Le cas des contrôleurs de la navigation aérienne dans le survol national. Unpublished Mémoire de DEA, Université de Toulouse le Mirail, Toulouse.

Button, G., \& Dourish, P. (1996). Technomethodology : paradox and possibilities. In Proceedings of the ACM conference on Human Factors in Computing Systemes, CHI'96. April 13-18. Vancouver, Canada: ACM.

Button, G., \& Sharrock. W. (1998). The Organizational Accountability of Technological Work. Social Studies of Science, 28(1), 73-102.

Cacciabue, C., Decortis, F., Drozdowicz, B., Masson, M., \& Nordvik, J. P. (1990). COSIMO : A Cognitive Simulation Model of Human Decision-Making and Behaviour in Complex Work Environments. Paper presented at the Second MOHAWC, Workshop.

Cardon, D. (1997). Les sciences sociales et les machines à coopérer. Une approche bibliographique du Computer Supported Cooperative Work. Réseaux, 85, 13-51.

Cefaï, D., \& Depraz, N. (2001). De la méthode phénoménologique dans la démarche ethnométhodologique. In M. de Fornel, A. Ogien, \& L. Quéré (Eds.), L'ethnométhodologie - Une sociologie radicale, Colloque de Cerisy. (pp. 99-119). Paris: La Découverte..

Clancey, W. J. (1997). Situated Cognition - On Human Knowledge and Computer Representations. Cambridge: Cambridge University Press.

Cognitive Science. (1993). Special issue: Situated Action. 17(1).

Conein, B. (1990). Cognition située et coordination de l'action - La cuisine dans tous ses états. Réseaux, 43, 99-110.

Conein, B. (1997). L'action avec les objets. Un autre visage de l'action située ? In B. Conein, \& L. Thévenot (Eds.), Cognition et Information en société ("Raisons Pratiques" 8) (pp. 25-45). Paris: EHESS.

Dourish, P. (1995). Accounting for system behavior : representation, reflection and resourceful action. In Proceedings of Computers in Context'95. Aarhus Denmark, August 1995.

Endsley, M. (1995). Toward a Theory of Situation Awareness. Human Factors, 37(1), 65-84.

Engeström, Y. (2000). From individual action to collective activity and back : developmental work research as an interventionist methodology. In P. Luff, J. Hindmarsh, \& C. Heath (Eds.), Workplace studies Recovering work practice and informing system design (pp. 150-166). Cambridge: Cambridge University Press.

Engeström, Y., Miettinen, R., \& Punamaki, R. L. (Eds.). (1999). Perspectives on Activity Theory. New York: Cambridge University Press.

Eriksen, S. (2002). Designing for accountability. In Proceedings of NordiCHI'02 (pp. 177-186). Arhus, Denmark, 19-23 October 2002: ACM.

Filippi, G., \& Theureau, J. (1995). Analyzing cooperative work in an urban trafic control rom for the design of a coordination support system. Paper presented at the Third European Conference on Computer-Supported Cooperative Work (ECSCW'95), Dordrecht, The Netherlands.

Fornel, M. de, Ogien, A., \& Quéré, L. (Eds.). (2001). L'ethnométhodologie - Une sociologie radicale, Colloque de Cerisy. Paris: La Découverte.

Garfinkel, H. (1967). Studies in Ethnomethodology. Oxford: Polity Press/Blackwell Publishers, 1984.

Goodwin, C., \& Goodwin, M. H. (1996). Seeing as a situated activity: Formulating planes. In Y. Engestrom, \& D. Middleton (Eds.), Cognition and Communication at work (pp. 61-95). Cambridge, USA: Cambridge University Press.

Grosjean, M., \& Lacoste, M. (1999). Communication et intelligence collective - Le travail à l'hôpital. Paris: PUF. 
Harper, R., \& Hughes, J. (1993). 'What a f-ing system! Send'em all to the same place and then expect us to stop'em hitting' Making technology work in air traffic control. In G. Button (Ed.), Technology in working order. Studies of work, interaction, and technology (pp. 127-144). London and NY: Routledge.

Harper, R., Hughes, J. A., \& Shapiro, D. (1989). The functionality of flight strips in ATC work (Report for the Civil Aviation Authority): Lancaster Sociotechnics Group, University of Lancaster.

Heath, C., \& Luff, P. (1992). Collaboration and control: Crisis management and multimedia technology in London Underground line control rooms. CSCW Journal, 1(1), 69-94.

Heath, C., \& Luff, P. (2000). Technology in action. Cambrige: Cambridge University Press.

Heath, C., Sanchez-Svensson, M., Hindmarsh, J., Luff, P., \& vom Lehn, D. (2002). Configuring awareness. Computer Supported Cooperative Work, Special issue on Awareness, 11, 317-347.

Heritage, J. (1984). Garfinkel and ethnomethodology. Cambridge: Polity Press.

Heritage, J. (1987). Ethnomethodology. In A. Giddens, \& J. Turner (Eds.), Social theory today (pp. 224-272). London: Polity Press (traduit partiellement dans Réseaux, 1991).

Heritage, J. (1990). Interactional accountability : a conversation analytic perspective. In B. Conein, M. de Fornel, \& L. Quéré (Eds.), Action analysis and conversation analysis (pp. 23-49). Paris: CENT (adaptation de Heritage 1988, Explanations as accounts: a conversation analytic perspective, In C. Hantaki (Ed.), Analysing lay explanation : a case book of methods, London: Sage).

Hughes, J. A., Randall, D., \& Shapiro, D. (1992). Faltering from Ethnography to Design. Paper presented at the Conference of Computer Supported Cooperative Work (CSCW 92), Toronto, Canada.

Hutchins, E. (1995). Cognition in the wild: Bradford Books-MIT Press.

Karsenty, L. (2002). Shifting the design philosophy of spoken natural language dialogue: from invisible to transparent systems. International Journal of Speech Technology, 5(2), 147-158.

Kirsh, D. (1995). The intelligent use of space. Artificial Intelligence, 72(1-2), 31-68.

Kuutti, K. (1996). Activity Theory as a potential framework for Human-Computer Interaction. In B. A. Nardi (Ed.), Context and Consciousness (pp. 17-44). Boston: MIT Press.

Latour, B. (1992). Where are the missing masses, Sociology of a few mundane artefacts. In W. Bijker, \& J. Law (Eds.), Shaping Technology-Building societies. Studies in sociotechnical change (pp. 225-259). Cambridge MA: MIT Press.

Leplat, J. (1991). Activités collectives et nouvelles technologies. Revue Internationale de Psychologie sociale, $4(3), 335-356$.

Leplat, J. (1997). Regards sur l'activité en situation de travail, contribution à la psychologie ergonomique. Paris: PUF.

Livet, P. (1994). La communauté virtuelle ; action et communication. Combas: Éditions de l'Éclat.

Livingstone, E. (1987). Making sense of ehtnomethodology. Londres: Routledge \& Kegan Paul.

Luff, P., \& Heath, C. (2000). The collaborative production of computer commands in command and control. International Journal of Human-Computer Studies (52), 669-699.

McCarthy, J., Wright, P., Healey, P., \& Harrison, M. (1997). Accountability of work activity in highconsequence work systems: Human error in context. International Journal of Human-Computer Studies (47), 735-766.

Mertz, C. (2003). Peripheral awareness offered by interaction techniques in Air Traffic control interfaces. In V. Belloti, T. Erikson, G. Cockton, \& P. Korhonen (Eds.), CHI 2003 New horizons Conference Workshop on "Providing elegant peripheral awareness", Fort-Lauderdale, April 5-10: ACM Press.

Norman, D. A. (1991). Cognitive artefacts. In J. M. Caroll (Ed.), Designing interaction psychology at the human-computer interface. Cambridge: Cambridge University Press.

Pavard, B., Benchekroun, T. H., \& Salembier, P. (1990). La régulation collective des communications : Analyse et Modélisation. In Actes du congrès Ergonomie et Intelligence Artificielle, ERGOIA'90, Biarritz.

Pougès, C., Guy, J., Pavard, B., Gourbeault, F., \& Champion, M. (1994). Conception de collecticiels pour l'aide à la prise de décision en situation d'urgence: la nécessité d'une approche pluridisciplinaire et 
intégrée. In B. Pavard (Ed.), Systèmes coopératifs: De la modélisation à la conception (pp. 351-375). Toulouse: Octarès Editions.

Quéré, L. (1997). La situation toujours négligée ? Réseaux(85).

Rognin, L., Salembier, P., \& Zouinar, M. (1997). Latent Organisational Reliability. In Proceedings of ALLFN'97 (pp. 63-71). Galway, Ireland: IEA PRESS.

Rognin, L., Salembier, P., \& Zouinar, M. (1998). Cooperation, Interactions and Socio-Technical Reliability: the case of Air-Traffic Control. Comparing French and Irish settings. In T. R. G. Green, L. Bannon, C. P. Warren, \& J. Buckley (Eds.), Proceedings of the European Conference on Cognitive Ergonomics (ECCE9).

Salas, E., Prince, C., Baker, D. P., \& Shrestha, L. (1995). Situation Awareness in Team Performance: Implications for Measurement and Training. Human Factors, 37(1), 123-136.

Salembier, P. (1996). Cognition(s) : Située, Distribuée, Socialement partagée, etc. (Bulletin du LCPE). Paris: ENS Ulm.

Salembier, P. (2002). Cadres conceptuels et méthodologiques pour l'analyse, la modélisation et l'instrumentation des activités coopératives situées. Systèmes d'information et Management (SIM), 7(2), 37-56.

Salembier, P., Kahn, J., Zorola-Villarreal, R., \& Zouinar, M. (1997). Assessing methods: Cognitive modeling (No. WP6, RHEA.): CEC, DG VII.

Salembier, P., \& Pavard, B. (2004). Analyse et modélisation des activités coopératives situées. Evolutions d'un questionnement et apports à la conception. @CTIVITES, 1(1). http://www.activites.org/

Salembier, P., Theureau, J., Zouinar, M., \& Vermersh, P. (2001). Action/Cognition située et assistance à la coopération. In J. Charlet (Ed.), Ingénierie des connaissances IC2001. Grenoble: PUG.

Savoyant, A. (1984). Définition et voies d'analyse de l'activité collective des équipes de travail. Cahiers de Psychologie Cognitive, 4(3), 273-284.

Schmidt, K. (1997). Of maps and scripts : the status of formal constructs in cooperative work. In Proceedings of GROUP'97, ACM Conference on Supporting Group Work, Phoenix, Arizona, 16-19 November 1997 (pp. 138-147). New-York, N.Y.: ACM Press.

Schmidt, K. (1998). Some notes on Mutual Awareness. Paper presented at the Special Interest Group on Mutual awareness, COTCOS-TMR, Paris, March 1998.

Schmidt, K. (2000). Distributed collective practices : a CSCW perspective. Paper presented at the Distributed Collective Practices conference, 19-22 septembre, Paris.

Schmidt, K. (2002a). The Problem with 'Awareness' - Introductory Remarks on 'Awareness in CSCW'. Computer Supported Cooperative Work, 11, 285-298.

Schmidt, K. (2002b). Remarks on the complexity of the cooperative work. In P. Salembier, \& T. H. Benchekroun (Eds.), Cooperation and Complexity, RSTIA. Paris-London: Hermes-Lavoisier (une version corrigée de ce texte est disponible à http://www.itu.dk/people/schmidt/.

Sperber, D., \& Wilson, D. (1995). Relevance. Communication and cognition (2nd edition ed.). Oxford: Basil Blackwell (première édition 1986, Cambridge, MA: Harvard University Press).

Stiegler, B. (1996). La technique et le temps 2. La désorientation. Paris: Galilée.

Suchman, L. (1983). Office procedures as practical actions: models of work and system design. $A C M$ Transactions on Office Information Systems, 1(4), 320-328.

Suchman, L. (1993). Technologies of Accountability: Of Lizards and Aeroplanes. In G. Button (Ed.), Technology in Working Order: Studies of Work, Interaction and Technology. London: Routledge.

Suchman, L. (1996). Constituting shared workspaces. In Y. Engestrom, \& D. Middleton (Eds.), Cognition and Communication at Work: Cambridge University Press.

Suchman, L., \& Wynn, E. (1984). Procedures and problems in the office. Office: Technology and People, (2), 133-154.

Terssac, G. de, \& Chabaud, C. (1990). Référentiel opératif commun et fiabilité. In J. Leplat, \& G. de Terssac (Eds.), Les facteurs humains de la fiabilité. (pp. 111-139). Marseille: Octarès 
Theureau, J., Filippi, G., Saliou, G., \& Vermersch, P. (2002). Cultural issues of nuclear power plant collective control in accidental situations and their impact upon design issues. In Proceedings of the Eleventh European Conference on Cognitive Ergonomics - ECCE 11. Catania, Italy, 8-11 september 2002.

Visetti, Y.-M. (1989). Compte rendu de Lucy A. Suchman, Plans and Situated Actions - The problem of Human/Machine Communication. Intellectica, 9.

Woods, D. D., Roth, E. M., \& Pople, H. (1987). Cognitive environment simulation : an artificial intelligent system for human performance assessment, Vol.2: Modeling human intention formation (NUREG-CR4862). Pittsburgh: Westinghouse.

Zouinar, M. (2000). Analyse et modélisation des processus de construction et d'actualisation du contexte partagé. Unpublished Thèse de Doctorat, CNAM, Paris.

Zouinar, M., \& Salembier, P. (2000). Modélisation du contexte partagé pour l'analyse et la conception des environnements de travail coopératif. In J. Charlet, M. Zackald, G. Kassel, \& D. Bourigault (Eds.), Ingénierie des connaissances - Evolution récentes et nouveaux défis (pp. 529-542). Paris: Eyrolles.

\section{RÉFÉRENCEMENT}

Salembier, P., \& Zouinar, M. (2004). Intelligibilité mutuelle et contexte partagé, Inspirations conceptuelles et réductions technologiques, @ctivités, 1 (2),64-85. http://www.activites.org/v1n2/salembier.pdf 